\title{
AN EXAMPLE OF A PLANE SHOCK OF VARIABLE STRENGTH
}

\author{
by P. SMITH \\ (Received 11th June 1963)
}

\begin{abstract}
A particular solution of the equations of one-dimensional anisentropic flow of a polytropic gas is linked by a shock to gas at rest in which the density is non-uniform. The approach is inverse in that the density distribution is derived from the position of the shock and the prescribed flow behind it. The velocity and strength of the shock each vary with time. The result is an example of the propagation of a shock through an inhomogeneous gas.
\end{abstract}

\section{Introduction}

Several examples of shocks of constant strength moving with variable velocity have been discussed previously by Copson (1) and Mackie and Weir (2). Although there occurs a jump in the entropy across the shock in these problems, the entropy remains uniform (but at different levels) on both sides of the shock: Thus the motion remains essentially homentropic, and the usual method of characteristics for homentropic flow can be applied.

More general situations, in which a shock leaves a non-uniform distribution of entropy behind it, usually require an approximate or numerical technique of solution. The present paper contains a comparatively simple example of such a flow. The solution described is purely illustrative; no general methods or applications are suggested. As is usual in the construction of solutions of this type, we start from known anisentropic flow and link this through a shock to gas at rest. The state of the stationary gas is not known until the shock path has been determined.

For the anisentropic flow behind the shock, we shall make the family of exact solutions discovered by Weir (3) our starting point. The main part of the paper concerns the matching of this flow to the stationary fluid so that the usual shock relations are satisfied.

\section{Weir's solution}

With viscosity and heat conduction neglected, the relevant equations for one-dimensional anisentropic flow are

$$
\rho_{t}+(\rho u)_{x}=0,
$$




$$
\begin{array}{r}
p_{x}+\rho u_{t}+\rho u u_{x}=0, \\
s_{t}+u s_{x}=0,
\end{array}
$$

where $p, \rho, s$ and $u$ are respectively pressure, density, specific entropy and fluid velocity. For a polytropic gas, the equation of state is

$$
p=K \rho^{\gamma} \exp \left(s / c_{v}\right),
$$

where $\gamma$ is the constant adiabatic index, $c_{v}$ the specific heat at constant volume and $K$ is a dimensional constant.

We shall merely state Weir's solution; the details can be seen in the original paper (3). It can be verified that equations (1) to (4) possess a solution

$$
\begin{aligned}
& u_{1}=2 \alpha t+\beta \text {, } \\
& p_{1}=f(X) \text {, } \\
& \rho_{1}=-\frac{1}{2 \alpha} \frac{d f}{d X}, \\
& s_{1}=c_{v} \ln \left\{(-2 \alpha)^{y} f \mid \frac{d f}{d X}\right\},
\end{aligned}
$$

where the particle paths are given by

$$
X=x-\alpha t^{2}-\beta t,
$$

and $X$ is the material variable. The suffix 1 is introduced to distinguish the flow behind the shock. We note certain arbitrary features in the unspecified constants $\alpha$ and $\beta$, and the function $f(X)$. The pressure and density are constant along the particle paths which are coaxial parabolas in the $(x, t)$-plane. The flow is one with constant acceleration.

\section{The shock path}

We shall now link the solution given in Section 2 to a state of rest through the usual shock relations for a polytropic gas, namely:

$$
\begin{aligned}
\rho_{01} v_{01} & =\rho_{2} v_{2}, \ldots \ldots \ldots \ldots . \\
p_{01}+\rho_{01} v_{01}^{2} & =p_{2}+\rho_{2} v_{2}^{2} \ldots \ldots . \\
\frac{\gamma}{\gamma-1} \frac{p_{01}}{\rho_{01}}+\frac{1}{2} v_{01}^{2} & =\frac{\gamma}{\gamma-1} \frac{p_{2}}{\rho_{2}}+\frac{1}{2} v_{2}^{2} .
\end{aligned}
$$

In equations (10), (11) and (12), $v$ is the velocity of the gas relative to the velocity of the shock and suffixes 01 and 2 are attached to the states immediately behind and in front (the stationary gas) of the shock respectively.

These shock relations must be satisfied at the shock whose displacement is taken as $\xi=\xi\left(t^{\prime}\right)$, where the prime has been added to the time $t$ since we shall subsequently use it as a parameter. From (9),

$$
X=\xi\left(t^{\prime}\right)-\alpha t^{\prime 2}-\beta t^{\prime},
$$


on the shock. It then follows from (5), (6) and (7) that

$$
\begin{aligned}
& u_{01}=2 \alpha t^{\prime}+\beta, \ldots \ldots \ldots \ldots \ldots \ldots \ldots \ldots \ldots \ldots \ldots \ldots \ldots \ldots \ldots \\
& p_{01}=g\left(t^{\prime}\right), \quad \ldots \ldots \ldots \ldots \ldots \ldots \\
& \rho_{01}=-\frac{\dot{g}\left(t^{\prime}\right)}{2 \alpha\left\{\dot{\xi}\left(t^{\prime}\right)-2 \alpha t^{\prime}-\beta\right\}}
\end{aligned}
$$

where

Furthermore

$$
g\left(t^{\prime}\right) \equiv f\left\{\xi\left(t^{\prime}\right)-\alpha t^{2}-\beta t^{\prime}\right\}
$$

$$
v_{01}=2 \alpha t^{\prime}+\beta-\dot{\xi}\left(t^{\prime}\right)
$$

In front of the shock, we take the gas to be at rest so that $u_{2}=0$ and $v_{2}=-\dot{\xi}\left(t^{\prime}\right)$. Further, $p_{2}=k$, a constant, since by equation (2) the pressure gradient must be zero.

The elimination of the unknown $\rho_{2}$ between (10) and (11), and (10) and (12) yields the following two equations:

$$
\begin{array}{r}
p_{01}-p_{2}=\rho_{01} v_{01}\left(v_{2}-v_{01}\right), \ldots \ldots \ldots . . . \\
2 \gamma\left(p_{01} v_{01}-p_{2} v_{2}\right)=(\gamma-1) \rho_{01} v_{01}\left(v_{2}^{2}-v_{01}^{2}\right) .
\end{array}
$$

Direct substitution in (17) and (18) of the flow quantities given previously produces

$$
\begin{array}{r}
\left(2 \alpha t^{\prime}+\beta\right) \dot{g}+2 \alpha g-2 \alpha k=0, \ldots \ldots \ldots \ldots \ldots \ldots . . . . . . . \\
\left(2 \alpha t^{\prime}+\beta\right)\left\{4 \gamma \alpha g-(\gamma-1)\left(2 \dot{\xi}-2 \alpha t^{\prime}-\beta\right) \dot{g}\right\}+4 \gamma \alpha(k-g) \dot{\xi}=0 .
\end{array}
$$

Solving the elementary differential equation (19), we have

$$
g=\frac{2 \alpha k t^{\prime}+A}{2 \alpha t^{\prime}+\beta}, \quad t^{\prime} \neq-\beta / 2 \alpha,
$$

where $A$ is a constant. The elimination of $g$ between (20) and (21) yields the shock velocity

where

$$
\dot{\xi}=B\left(2 \alpha t^{\prime}+\beta\right)^{2}+\frac{1}{2}(\gamma+1)\left(2 \alpha t^{\prime}+\beta\right),
$$

$$
B=k \gamma /(A-k \beta) \text {. }
$$

Integration of (22) provides the equation of the shock path, namely

$$
\xi=\frac{B}{6 \alpha}\left(2 \alpha t^{\prime}+\beta\right)^{3}+\frac{\gamma+1}{8 \alpha}\left(2 \alpha t^{\prime}+\beta\right)^{2}+C,
$$

where $C$ is a further constant. Thus the shock relations determine the shock path and fix the flow quantities on both sides of the shock. In terms of the spatial variables, the pressure and density behind the shock are given parametrically by

$$
p_{1}=\frac{2 \alpha k t^{\prime}+A}{2 \alpha t^{\prime}+\beta}
$$




$$
\begin{aligned}
& \rho_{1}=\frac{A-k \beta}{\left(2 \alpha t^{\prime}+\beta\right)^{3}\left\{B\left(2 \alpha t^{\prime}+\beta\right)+\frac{1}{2}(\gamma-1)\right\}}, \\
& x-\alpha t^{2}-\beta t=\xi\left(t^{\prime}\right)-\alpha t^{\prime 2}-\beta t^{\prime}, \quad \ldots \ldots \ldots . .
\end{aligned}
$$

where (24) and (25) follow from (15) and (16), whilst (26) is obtained by eliminating $X$ between (9) and (13).

For the stationary gas in front of the shock the pressure is uniform and the density is given parametrically by

$$
\begin{aligned}
\rho_{2} & =\frac{A-k \beta}{\left(2 \alpha t^{\prime}+\beta\right)^{3}\left\{B\left(2 \alpha t^{\prime}+\beta\right)+\frac{1}{2}(\gamma+1)\right\}} \\
x & =\xi\left(t^{\prime}\right) . \ldots \ldots \ldots \ldots \ldots \ldots \ldots \ldots \ldots \ldots \ldots \ldots \ldots \ldots \ldots \ldots \ldots \ldots
\end{aligned}
$$

The calculation of $\rho_{2}$ from (10) produces the density distribution as a function of time on the shock path, namely equation (27). To obtain the density as a function of $x$, we eliminate $t^{\prime}$ between (27) and the equation (28) of the shock path. Finally, the entropy of the stationary gas can then be derived from the equation of state (4) as a function of $x$.

A general point is worth noting here. It can be verified that equations (1) to (4) have a solution

$$
u=0, \quad \rho=\rho(x), \quad p=\text { constant }
$$

in which the entropy is also a function of $x$ determined by the equation of state. Such a situation is possible in a perfect gas. In a real fluid, the influence of, for example, thermal conduction would operate against the equilibrium of this state.

\section{Properties of the solution}

The formal solution is now complete. Essentially, we have found a family of solutions whose members depend on the choice of values for the various constants. However, we must add certain physical requirements. We shall simplify the ensuing discussion by assuming $\alpha>0, \beta>0$ and restricting our attention to $t \geqq 0$. The shock must be compressive, that is, $0<p_{2}<p_{01}$, or

$$
0<k<\frac{2 \alpha k t^{\prime}+A}{2 \alpha t^{\prime}+\beta}, \quad\left(t^{\prime} \geqq 0\right),
$$

by (24). Thus $A-k \beta>0$ and all pressures are positive. Inspection of (25) and (27) indicates that the densities are positive if $\gamma>1$.

Taking $p_{01} / p_{2}$ as a measure of the strength of the shock, we have

$$
\mu=\frac{p_{01}}{p_{2}}=\frac{2 \alpha k t^{\prime}+A}{k\left(2 \alpha t^{\prime}+\beta\right)} .
$$

The shock strength has a maximum at $t^{\prime}=0$, and $\mu \rightarrow 1$ as $t^{\prime} \rightarrow \infty$ indicating that the shock becomes infinitely weak. The shock velocity $\dot{\xi} \rightarrow \infty$ as $t^{\prime} \rightarrow \infty$ from (22). 
At $t=0, \rho_{2} \rightarrow 0$ as $x \rightarrow \infty$. Since the temperature $T_{2} \propto p_{2} / \rho_{2}$, the latter statement is equivalent to an unbounded increase in the temperature.

The total effect is that of a shock accelerating into a gas possessing a negative temperature gradient.

Fig. 1 shows a typical configuration of the shock path and particle

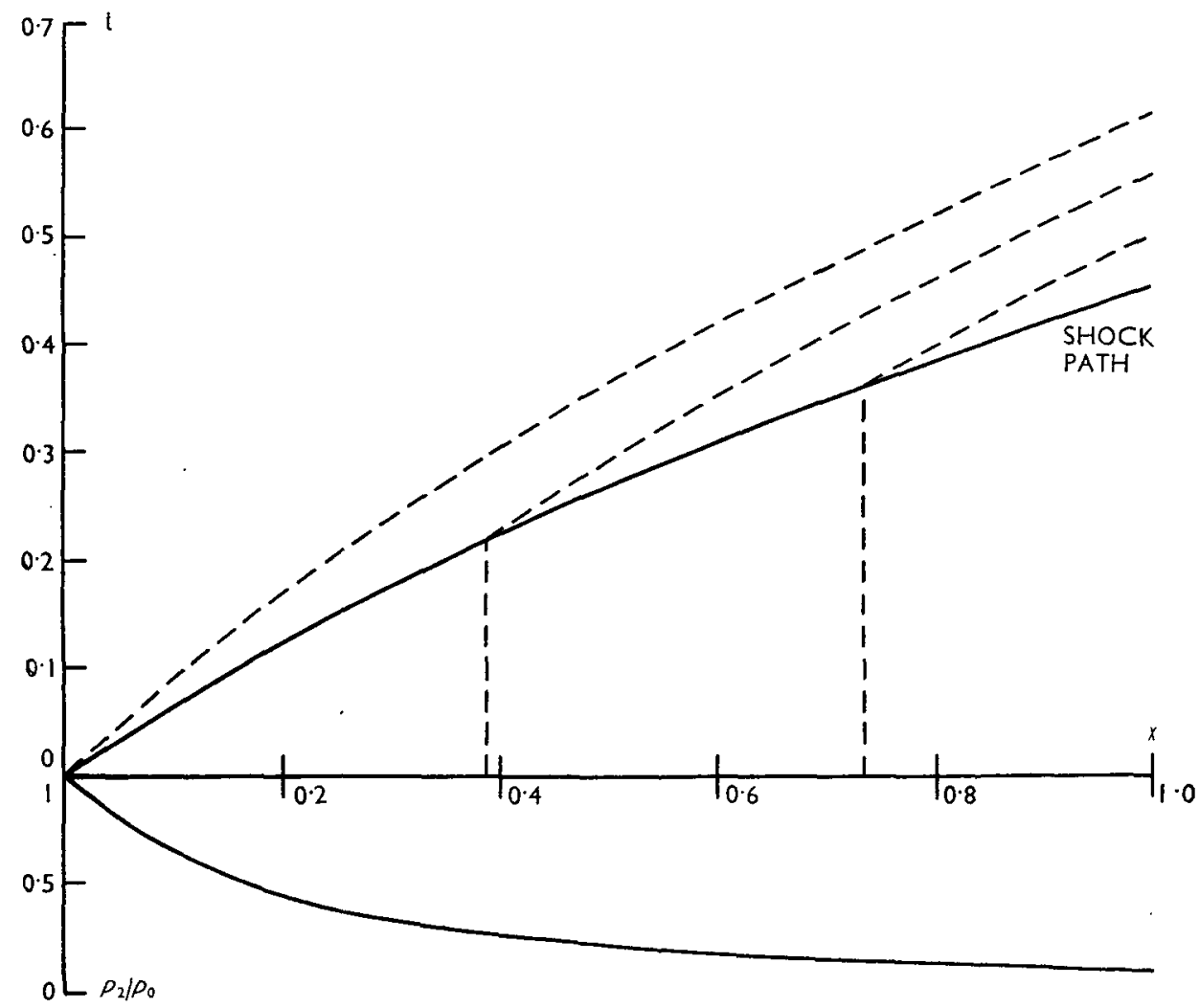

FIG. 1

trajectories in the $(x, t)$-plane. The lower part of the figure gives the density ratio in the gas upstream of the shock; $\rho_{0}$ is the density at $x=0, t=0$. The values chosen for the various constants are as follows:

$$
\alpha=\beta=1 ; B=\frac{1}{2}(\gamma-1) ; C=-\frac{5 \gamma+1}{24} ; \gamma=\frac{7}{5} \text {. }
$$

The motion can be considered as taking place in an infinite tube closed at one end by a piston. At time $t=0$ from $x=0$, the piston is pushed into the gas with displacement given by the particle trajectory which passes through the origin. The initial motion of the piston is impulsive. 


\section{Uniform compressive motion}

Generally, the more interesting and physically reasonable problems are those in which the gas ahead of the shock is at rest with constant density and temperature. From (27) this will be so only if $\alpha=0$. The particle trajectories behind the shock are then a family of straight lines with fluid velocity $u=\beta$. The shock velocity is also constant and is given by

from (22).

$$
\dot{\xi}=B \beta^{2}+\frac{1}{2}(\gamma+1) \beta,
$$

The special motion presented above is simply the uniform compressive motion described by Courant and Friedrichs (4), where the shock velocity is found to be

$$
\dot{\xi}=\frac{1}{4}(\gamma+1) u_{1}+\sqrt{ }\left\{c_{2}^{2}+\frac{1}{16}(\gamma+1)^{2} u_{1}^{2}\right\},
$$

with $c_{2}$ denoting the sound speed upstream of the shock. In our notation

$$
u_{1}=\beta, \quad c_{2}=\sqrt{ }\left(\frac{\gamma p_{2}}{\rho_{2}}\right)=\sqrt{ }\left\{B \beta^{3}\left\{B \beta+\frac{1}{2}(\gamma+1)\right\}\right],
$$

from equations (23) and (27). Equation (29) can be recovered by substituting for $u_{1}$ and $c_{2}$ in (30). This provides a partial check on our family of solutions.

\section{REFERENCES}

(1) E. T. Copson, Proc. Roy. Soc. A222 (1954), 254-261.

(2) A. G. MACKIE and D. G. WeIR, Proc. Camb. Phil. Soc., 56 (1960), 64-74.

(3) D. G. Weir, Proc. Camb. Phil. Soc., 57 (1961), 890-894.

(4) R. Courant and K. O. FrIEdrichs, Supersonic Flow and Shock Waves (New York, 1948), p. 150.

\section{Department of Mathematics}

THE UNIVERSITY

KEELE

STAFFORDSHIRE 